\title{
Factores explicativos de la demanda municipal de agua y efectos en el bienestar de la política tarifaria. Una aplicación a la cuenca del Segura
}

\author{
Miguel Ángel TobARRA-GONZÁLEz \\ Universidad Politécnica de Cartagena, Facultad de CC.EE., c/ Real, 3, 30201 Cartagena, España. E- \\ mail: miguel.tobarra@upct.es
}

\begin{abstract}
RESUMEN
En este artículo se analiza la demanda urbana de agua en alta a partir de datos mensuales de 42 municipios situados en la cuenca del Segura, para el período 2000-2006. La estimación econométrica realizada permite afirmar que el precio, la renta y la población son las variables que mayor efecto tienen en la demanda urbana de agua. Su comportamiento es inelástico, con una elasticidad precio de $-0,4$, característica de un bien necesario con pocos sustitutivos. De las simulaciones realizadas se desprende que incrementos de la tarifa que consiguieran reducir el consumo un $10 \%$ se traducirían en reducciones del excedente del consumidor de entre 8 y 9,5 euros por persona. Asimismo, existe un mínimo de consumo de agua en el hogar, 84 litros por habitante y día, que no se verá afectado por subidas tarifarias. Las variables renta y población tienen un efecto más significativo que el precio para explicar variaciones en la demanda. Por ello, las previsiones acerca de la evolución de estas variables son claves para una adecuada gestión hídrica.
\end{abstract}

Palabras clave: Demanda urbana de agua, política tarifaria.

\section{Explicative Factors of Municipal Water Demand and Effects of Tariff Policy on Welfare. The Case of Segura Basin}

\begin{abstract}
In this article urban water demand is analyzed using data of 42 municipalities located in the Segura River Basin for the period 2000-2006. The econometric approach shows that price, income and population are the most important variables for explaining urban water demand changes. Water tariffs achieve moderate savings due to the price inelastic behavior of demand $(-0,4)$. This is typical of necessary goods without substitutes. Calculus made shows that the increment in tariffs, necessary to reduce $10 \%$ consumption, means a consumer surplus reduction between 8 and 9,5 euros per person (and year). There is also a minimum of water consumption in homes, 84 litres per capita per day that cannot be affected by tariffs. Income and population increments have deeper impact than price for explaining changes in urban water demand. Predictions about these variables are major for a proper hydraulic management.
\end{abstract}

Keywords: Urban Water Demand, Tariff Policy.

Clasificación JEL: H4,Q21,Q25

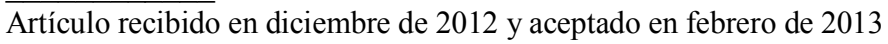

Artículo disponible en versión electrónica en la página www.revista-eea.net, ref. ə-31202 


\section{INTRODUCCIÓN}

El agua es un recurso vital además de un input clave para la producción de numerosos bienes y su escasez puede ser un factor limitante para el desarrollo nacional (Pérez, Gómez y Garrido 2010). Aunque es un recurso renovable, el crecimiento de la población, el incremento en el uso económico y energético y los posibles cambio climatológicos podrían generar importantes problemas de escasez y conflictividad en el futuro (Sevilla, Torregrosa y Moreno 2010). Para una gestión adecuada de un recurso natural como el agua, sujeto a múltiples usos, el análisis económico es un instrumento cada vez más necesario, máxime desde la aprobación de la Directiva Marco del Agua en Europa (Gómez-Limón y Martín-Ortega 2011).

El estudio del suministro urbano de agua es fundamental pues se trata de un uso prioritario. Sin embargo, los estudios académicos de la demanda urbana en España son escasos. Cabría citar, entre otros, los trabajos de Trujillo (1994), Martínez Espiñeira (2003, 2007), Arbués, Barberán y Villanúa (2004), García Valiñas (2005), Barberán, Costa y Alegre (2008), Beamonte, Casino y Veres (2010) y Del Villar (2010). Trujillo (1994) se centra en el municipio de Las Palmas de Gran Canaria. A partir de datos de consumo a nivel agregado y costes del servicio para los años 1987-1991, proporcionados por el suministrador, trata de medir los cambios que se producirían en el bienestar de los consumidores si se pasara de la tarifa con bloques crecientes aplicada en el municipio a una lineal. Martínez Espiñeira (2003) utiliza datos de tres municipios del noroeste español para contrastar la especificación de precios sugerida por Nordin (1976) en la estimación de funciones de demanda de agua, aplicable en el caso de tarifas por bloques crecientes. Martínez Espiñeira (2007), a partir de datos anuales de uso de agua en el hogar en la ciudad de Sevilla, para el periodo 1991-1999, obtiene elasticidades precio de la demanda a corto (-0.1) y a largo plazo (-0.5) en esta ciudad. Arbués, Barberán y Villanúa (2004) analizan el impacto de las tarifas de agua en el consumo urbano de la ciudad de Zaragoza. Para el mismo municipio, Barberán, Costa y Alegre (2008) realizan un estudio de la recuperación de costes de este servicio. García Valiñas (2005), a partir de datos de consumo en hogares del municipio de Elche, para el periodo 19942000, analiza el incremento del bienestar que implicaría el uso de los esquemas de Ramsey y Feldstein en las tarifas del agua. Beamonte, Casino y Veres (2010) estudian indicadores de la calidad del agua y la relación entre ésta y el gasto por su consumo en los hogares. Del Villar (2010) realiza un ejercicio de simulación con el que prevé los efectos sobre el consumo de la traslación a precios de los costes de las inversiones previstas en la planificación hidrológica. Además, cabe señalar las revisiones bibliográficas de Arbués, García Valiñas y Martínez Espiñeira (2003) y de González Gómez y García Rubio (2008), relativas a la estimación de la demanda de agua residencial y la eficiencia en la gestión del 
servicio urbano de agua respectivamente.

El proceso de abastecimiento urbano consta de tres fases, la primera sería la captación del recurso del medio y su tratamiento previo para garantizar que se den las condiciones de potabilidad adecuadas, denominada suministro en alta o primario. A continuación se daría una segunda fase de distribución para hacer llegar el agua a los puntos de consumo (los hogares). Finalmente, el agua es recogida por las redes de alcantarillado tras su uso y debe ser convenientemente tratada para devolverla al medio en las condiciones en las que fue captada.

Así, la demanda que hacen los ayuntamientos para, seguidamente, distribuir el agua por sus redes, constituye la demanda en alta del recurso. El trabajo aquí presentado es novedoso porque, hasta donde el autor conoce, se trata del primer estudio de demanda de agua en alta que se realiza en nuestro país. Además, cuenta con una muestra importante, 42 municipios de la Comunidad Autónoma de la Región de Murcia, cuando la mayoría de los estudios realizados en España se centran en un solo municipio. Los objetivos de este trabajo son fundamentalmente dos. En primer lugar, conocer los factores que determinan la demanda municipal de agua. En segundo lugar, conocer la efectividad en la reducción del consumo y los efectos sobre el bienestar de los ciudadanos de la política tarifaria.

El artículo se estructura de la siguiente manera. En la sección dos se describe la cuenca del Segura y el servicio municipal de agua en esta demarcación. En la sección tres se analizan los factores explicativos de la demanda municipal de agua y se exponen los datos y la metodología utilizada. En la sección cuatro se presentan los principales resultados. En la sección cinco se realiza una simulación de los efectos de la política tarifaria en el bienestar de los ciudadanos. En la sección seis se calcula el límite inferior de consumo en el hogar que no puede ser afectado por la política tarifaria. Finalmente, en la sección siete se presentan las principales conclusiones.

\section{DESCRIPCIÓN DE LA CUENCA DEL SEGURA}

La Demarcación Hidrográfica del Segura se encuentra en el sureste de España. Con una superficie aproximada de $18.870 \mathrm{~km}^{2}$ supone un 3,7\% del territorio español. Afecta a cuatro comunidades autónomas: en su totalidad a la de Murcia y parcialmente a las comunidades de Andalucía (provincias de Jaén, Granada y Almería), Castilla-La Mancha (provincia de Albacete) y Valencia (provincia de Alicante). Su población de derecho en 2006 era de 1.850 .000 habitantes, cerca del $4,14 \%$ del total nacional. Presenta un clima mediterráneo con precipitaciones escasas e irregulares.

La evolución temporal de las demandas muestra que, tras un crecimiento histórico continuado y acelerado en los años setenta y ochenta por la construcción del trasvase Tajo-Segura, la superficie de regadío se muestra estable, 
mientras que existe un crecimiento continuado de la demanda urbana.

La mayor parte del suministro urbano en el ámbito territorial de la cuenca del Segura se lleva a cabo por la Mancomunidad de Canales del Taibilla (MCT en adelante), que sirve los recursos tratados a los depósitos en alta para su distribución por las redes municipales. Esta entidad gestiona unos $200 \mathrm{hm}^{3}$ al año, tanto para el suministro a población de la cuenca del Segura como del Júcar.

Así, se puede estimar que cerca de un $77 \%$ del volumen gestionado por la MCT es destinado a la Demarcación del Segura, mientras que el 23\% restante se destina a la Demarcación del Júcar.

Por municipios, la Mancomunidad suministra a 76 municipios de los que 32 corresponden a la Comunidad Valenciana, 42 a la Comunidad Autónoma de la Región de Murcia y dos a Castilla la Mancha. Estos municipios suponen una población abastecida de dos millones de habitantes, cifra que se incrementa en 800.000 más en la época estival.

Para proporcionar este servicio, la Mancomunidad cuenta como fuente principal con los recursos del río Taibilla y los recursos del acueducto Tajo-Segura $\mathrm{y}$, secundariamente, con otros volúmenes procedentes de desalación y aguas subterráneas.

\section{ANÁLISIS DE LOS FACTORES EXPLICATIVOS DE LA DEMANDA MUNICIPAL DE AGUA}

Las variables explicativas que suelen emplearse en los modelos de consumo urbano de agua son el precio, la renta y otras variables indicadoras de clima y características del hogar. A continuación se describen las variables utilizadas en el modelo econométrico que se presenta en esta sección.

La variable dependiente será la cantidad de $\mathrm{m}^{3}$ de agua suministrada mensualmente en alta a los ayuntamientos por la $\mathrm{MCT}^{1}$.

Entre las variables independientes o explicativas se utilizarán el precio, la renta per cápita de cada ayuntamiento, tres variables demográficas (población, número de viviendas secundarias y número de pernoctaciones), dos variables climáticas (temperatura y precipitación), una variable relativa a la estructura de la población (porcentaje de la población menor de 25 años) y otra relativa al impacto de la industria conectada a redes municipales. Hay que señalar que aunque variables de este tipo han sido usadas en la literatura, ningún estudio de los revisados por el autor incluye simultáneamente todas éstas. Seguidamente, se describen con detalle.

El precio es la tarifa que cobra la MCT por $\mathrm{m}^{3}$ suministrado, que es la misma

\footnotetext{
${ }^{1}$ El autor quiere agradecer la colaboración de esta entidad, que ha proporcionado los datos de suministro de agua en alta a los ayuntamientos recogidos en este estudio.
} 
para todos los municipios que abastece. Asimismo éste no varía en función del consumo, todas las unidades tienen el mismo precio, sin que haya tampoco ningún componente fijo. Por tanto, precio medio y marginal coinciden plenamente. Esto hace innecesario tener que comprobar a qué precio de los dos anteriores responde la demanda. Al mismo tiempo, la no existencia de componentes fijos ni de tarifas por tramos crecientes facilita el cálculo econométrico ${ }^{2}$. Las tarifas cobradas por la MCT han sido deflactadas utilizando el Índice de Precios al Consumo de la Región de Murcia. Por tanto, se manejan tarifas en términos reales.

Como variable renta se utiliza la renta bruta disponible per cápita de cada municipio $^{3}$. Es de destacar que en este trabajo se utilizan auténticos datos de renta, y no la proxy habitual en estudios de consumo urbano de agua de valor de la vivienda como aproximación de la renta del hogar (lo que no permite cambios en la renta a lo largo del tiempo). Los datos a nivel municipal sólo están disponibles para el periodo 2000-2006. La renta bruta disponible es un dato anual que se transforma en datos mensuales al dividir por doce la cuantía anual.

Sin duda las variables demográficas son importantes. A este efecto habrá una clara relación positiva entre agua demandada y población del municipio. De hecho, las previsiones de los planes de cuenca sobre evolución futura de la demanda se basan en las predicciones demográficas elaboradas por el Instituto

${ }^{2}$ La existencia de tarifas con tramos conlleva un problema de endogeneidad entre la variable explicada, el consumo de agua, y la variable explicativa, el precio, ya que en este caso se determinarían mutuamente. Desde hace tiempo se han venido proponiendo métodos para tratar de solventar este problema en el cálculo econométrico. De hecho, una de las líneas en las que más se ha investigado en los últimos años es la relativa a la estimación de la demanda en presencia de tramos con precios crecientes y componentes fijos en la tarifas hídricas. Así, se pueden mencionar, entre otros, los trabajos de Bell y Griffin (2008, 2011), Martínez-Espiñeira (2003), Olmstead et al., (2007) y Taylor, McKean y Young (2004).

Asimismo, no hay unanimidad en la literatura acerca de cuál es el precio al que responde el consumidor, el precio medio o el marginal. Shin (1985) propone un modelo de percepción del precio con un parámetro $\mathrm{k}$ que indicaría cuál es el precio percibido por el consumidor, ante el que éste reaccionaría. Arbués, Barberán y Villanúa (2004) indican que dada la complejidad de las tarifas en Zaragoza, la variable precio a la que mejor responden los ciudadanos es un precio medio retardado obtenido a partir de la factura de agua. Bell y Griffin (2008), con datos de suministradores de agua de Texas, señalan que el cambio en el precio marginal del agua explica mejor el consumo que el cambio en el precio medio.

${ }^{3}$ Estos datos se han obtenido del estudio del Consejo Económico y Social de la Región de Murcia, "Estimación de la renta bruta disponible de los hogares de los municipios de la Región de Murcia durante el periodo 2000-2006. Análisis de las disparidades intermunicipales de renta y bienestar" (2010). Lamentablemente no se dispone de una serie de datos superior. Las estimaciones de la renta disponible que realiza el INE sólo están desagregadas a nivel de provincia. El anuario económico de España elaborado por la Caixa sólo presenta un indicador de renta municipal consistente en una cuota de mercado que reflejaría el poder adquisitivo del conjunto de cada municipio sobre una base de 100.000 unidades. 
Nacional de Estadística (INE en adelante). Los datos se han obtenido del Padrón Municipal de Habitantes.

Asimismo, no hay que olvidar el componente de población turística. Parte de este turismo es de segunda residencia, cuyo efecto se recoge a través de la variable viviendas secundarias ${ }^{4}$. Para recoger el resto se utilizará la variable número de pernoctaciones mensuales por municipio 5 .

Los efectos climáticos se han introducido en la literatura de diferentes formas. Con indicadores de precipitación, evapotranspiración, temperatura o una combinación de ellos. Como indicadores climáticos se utilizará precipitación (litros por $\mathrm{m}^{2}$ ) y la temperatura media mensual (en grados centígrados) en cada municipio $^{6}$.

Previsiblemente la demanda de agua tendrá un componente estacional en los meses de verano, derivado de dos efectos, la mayor temperatura y el turismo.

También se utiliza un indicador de la estructura demográfica pues hogares con más miembros jóvenes suelen presentar mayores consumos de agua. Por ello, se incluye el porcentaje de población menor de 25 años en cada municipio $^{7}$.

Finalmente, se introducirá una variable que recoja la influencia del sector industrial en el consumo de agua de la población. Para ello se utilizará el número de empleos en este sector ${ }^{8}$. El Plan Hidrológico de la cuenca del Segura estima que aproximadamente la mitad del consumo de agua industrial corresponde a pequeña industria conectada a las redes municipales. Esto significaría que aproximadamente el $12 \%$ del suministro urbano de agua tendría como destino pequeñas industrias conectadas a las redes municipales.

${ }^{4}$ Estos datos se han elaborado a partir de la información contenida en el Censo de vivienda de 2001 y los datos acerca de viviendas terminadas obtenidos de la publicación Municipios en cifras de la Región de Murcia, elaborada por el Centro regional de estadística de Murcia.

${ }^{5}$ Los datos de pernoctaciones son publicados por la Consejería de Cultura y Turismo de la Comunidad Autónoma de la Región de Murcia. Sólo están disponibles a nivel municipal para los municipios de Murcia, Cartagena, Mazarrón y Águilas. El resto vienen agregados en seis zonas, Lorca/Puerto Lumbreras, Resto del Mar Menor, Noroeste, Valle de Ricote/Balnearios, Centro y Altiplano Este. En este trabajo, se ha optado por distribuir el número de pernoctaciones de cada zona entre los municipios que lo forman, ponderando la distribución con la población de cada uno de ellos.

${ }^{6}$ Los datos de precipitación y temperaturas por municipios vienen recogidos en la publicación Territorio, Climatología y Medio Ambiente, elaborada por el Centro regional de estadística de Murcia. A su vez, éstos son facilitados por la Agencia estatal de meteorología, Centro Metereológico Territorial de Murcia.

${ }^{7}$ Elaborado a partir de los datos del Padrón Municipal de Habitantes.

${ }^{8}$ Se trata de un dato anual recogido en el Anuario Estadístico de la Región de Murcia elaborado por el Centro regional de estadística de Murcia. 
Con el objeto de comprender la importancia de cada una de las variables explicativas en la determinación de la demanda de agua se utilizará un modelo log log en la estimación econométrica. Los coeficientes así estimados serán elasticidades de la demanda de agua en relación a cada uno de los parámetros. La estimación econométrica se hará por mínimos cuadrados ordinarios adaptados a datos de panel con el programa E-views.

El modelo a estimar será:

$\ln \mathrm{CONSUMO}_{\text {it }}=$

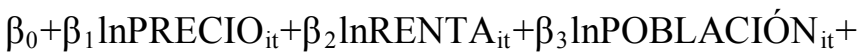

$+\beta_{4} \operatorname{lnVIVSEC}_{\mathrm{it}}+\beta_{5} \ln _{\text {nERNOCTACIONES }}+\beta_{6} \operatorname{lnTEMPERATURA}_{\mathrm{it}}+$

$+\beta_{7} \operatorname{lnPRECIPITACIÓN} \mathrm{it}_{\mathrm{it}}+\beta_{8} \operatorname{lnPOBLA} 25_{\mathrm{it}}+\beta_{9} \ln$ INDUSTRIA $_{\mathrm{it}}+\mathrm{e}_{\mathrm{it}}$

con $\mathrm{i}=1 \ldots 42$ y $\mathrm{t}=1 \ldots 84$, donde:

- $\beta_{0, \ldots,} \beta_{9}$ son los coeficientes a estimar

- CONSUMO es el número de $\mathrm{m}^{3}$ consumidos mensualmente en cada municipio

- PRECIO, la tarifa por $\mathrm{m}^{3}$ de agua cobrada por MCT, deflactada con el Índice de Precios al Consumo de la Región de Murcia

- RENTA, la renta bruta disponible per cápita mensual de cada municipio

- POBLACIÓN, habitantes del municipio

- VIVSEC, el número de viviendas secundarias del municipio

- PERNOCTACIONES, el número mensual de pernoctaciones en cada municipio

- TEMPERATURA, la temperatura media mensual en cada municipio

- PRECIPITACIÓN, la precipitación mensual en cada municipio

- POBLA25, el porcentaje de población menor de 25 años

- INDUSTRIA, número de empleos en la industria de cada municipio

- e, el término de error

Presentado el modelo a estimar, la próxima sección explica los principales resultados obtenidos.

\section{RESULTADOS PRINCIPALES}

Los resultados de la estimación del modelo [1] aparecen recogidos en la Tabla resumen del anexo. Sólo la variable población menor de 25 años no es significativa por lo que se considera una especificación del modelo en la que se excluye esta variable. La estimación de esta especificación se presenta a continuación en la Tabla 1. 
Tabla 1

Estimación del modelo [1]

\begin{tabular}{|c|c|c|c|c|}
\hline Variable & Coeficiente & Error estándar & Estadístico t & Prob \\
\hline C & -2.849738 & 0.357183 & -7.978370 & 0.0000 \\
\hline PRECIO & -0.408351 & 0.083422 & -4.895031 & 0.0000 \\
\hline RENTA & 0.886609 & 0.046609 & 19.02243 & 0.0000 \\
\hline POBLACION & 0.645215 & 0.017745 & 36.35965 & 0.0000 \\
\hline VIVSEC & 0.041292 & 0.005198 & 7.944125 & 0.0000 \\
\hline PERNOCTACIONES & 0.045423 & 0.007547 & 6.018406 & 0.0000 \\
\hline TEMPERATURA & 0.266664 & 0.019472 & 13.69460 & 0.0000 \\
\hline PRECIPITACION & -0.020664 & 0.002417 & -8.548064 & 0.0000 \\
\hline INDUSTRIA & 0.111208 & 0.009357 & 11.88441 & 0.0000 \\
\hline Indicadores de bondad de ajuste & \\
\hline R-cuadrado & 0.906259 & Criterio Akaike & 0.955076 \\
\hline R-cuadrado Ajustado & 0.906046 & \multicolumn{2}{|c|}{ Criterio Schwarz } & 0.970812 \\
\hline Log verosimilitud & -1675.754 & \multicolumn{2}{|c|}{ Prob(F-estadístico } & 4252.594 \\
\hline Estadíst. Durbin-Watson & 0.155934 & \multicolumn{5}{c|}{0.000000} \\
\hline
\end{tabular}

Fuente: Elaboración propia.

Todas las variables presentan los signos esperables a priori. Una relación negativa entre consumo de agua y precio y precipitación, y positiva para el resto. De forma conjunta contribuirían a explicar el $90 \%$ de la variación en el consumo de agua.

El coeficiente estimado para la variable precio se interpreta como la elasticidad de la demanda con respecto al precio. El valor obtenido indica que un incremento de la tarifa del $10 \%$ conseguiría un descenso en la demanda de agua del $4 \%$. Por tanto, la política tarifaria es una herramienta a través de la cual se puede reducir el consumo de agua si bien tiene un impacto moderado. Esto no es sorprendente dado que el agua es un bien necesario sin productos sustitutivos, al menos a corto plazo (a largo plazo podría haber modificaciones en las instalaciones encaminadas a reducir el agua necesaria).

El coeficiente estimado para la variable renta se interpreta como la elasticidad renta de la demanda. Un incremento de la renta de un $10 \%$ incrementaría el consumo del agua en un $8,8 \%$. Por tanto, el crecimiento económico parece llevar aparejado un mayor consumo de agua. Se hace necesario tomar medidas que compatibilicen crecimiento económico y conservación del recurso.

El coeficiente estimado para la variable población mide la sensibilidad de la demanda de agua al aumento demográfico. Así, un incremento de la población de un $10 \%$ aumentaría la demanda de agua un $6,45 \%$. La existencia de economías de escala en el consumo de agua en el hogar podría explicar por qué los 
incrementos poblacionales no se traducen en incrementos de la demanda de agua de la misma proporción.

El efecto de la población turística viene recogido por las variables vivienda secundaria, que trata de recoger el turismo de segunda residencia, y número de pernoctaciones. El efecto de ambas variables es prácticamente el mismo. Por tanto, no habría diferencias significativas en el consumo de agua entre un tipo de turismo y otro. Un incremento del turismo de la cuenca del $10 \%$ se traduciría en un aumento de casi el 1\% de la demanda de agua de toda la cuenca. No obstante, éste es un dato medio y sería superior para aquellos municipios con un marcado carácter turístico.

Incrementos de la temperatura también tienen una repercusión positiva en la demanda urbana de agua. No obstante, el impacto es moderado aunque podría no ser lineal. Previsiblemente tendría un impacto más grave en la demanda agraria, consumidora de un volumen de agua mucho mayor.

La precipitación no reduce significativamente el consumo municipal de agua. El coeficiente asociado a esta variable es el más reducido de todos los estudiados. Un factor explicativo pudiera ser que el consumo del hogar en el exterior sea más reducido en el caso de España que en otros países debido al modelo urbanístico, en el que predomina el piso sobre la vivienda con jardín. Cambios en esta tipología aumentarían la demanda urbana de agua y la harían más sensible a las precipitaciones.

La evolución de la demanda de consumo industrial es difícil de prever pues depende de factores como la evolución de la producción o del tipo de industria asentada en un lugar, y en ocasiones, de fenómenos puntuales como el establecimiento de grandes empresas. No obstante, y con la debida prudencia, para la Región de Murcia, el modelo señala que un incremento del 10\% del empleo industrial se traduciría en un incremento de algo más del $1 \%$ en la demanda de agua de los ayuntamientos.

Dado que se dispone de una sección cruzada de 42 municipios, cabe pensar en la posibilidad de particularidades locales en el consumo. Se trataría de efectos latentes no observables, específicos de cada municipio; una suerte de idiosincrasia local que incidiría en el consumo de agua. Sin embargo, el ámbito al que pertenecen, la cuenca del Segura, con unas características climáticas y culturales muy similares, hace pensar que, caso de producirse, esos efectos no serían muy significativos. La Tabla resumen del anexo recoge esta posibilidad con los resultados de un modelo de efectos aleatorios ${ }^{9}$ (MEAL) y un modelo de

\footnotetext{
${ }^{9}$ En el modelo MEAL se considera una variable latente, propia de cada localidad, que se distribuiría con media cero y varianza finita. En esta estimación se utilizan mínimos cuadrados generalizados factibles siguiendo la metodología recogida en Baltagi (2001).
} 
efectos fijos ${ }^{10}$ (MEF). Las mismas permiten mantener los resultados ya comentados en cuanto a la importancia relativa de las variables en la explicación del consumo municipal de agua. Por tanto, los resultados obtenidos son robustos.

Dado que el panel es de sólo siete años se prescinde del estudio de estos efectos en su dimensión temporal.

Asimismo, se puede considerar que el consumo de agua tiene un componente estacional. Los meses de verano muestran un pico en el consumo y los de invierno un valle. Por ello se incluye en la Tabla resumen los resultados de un modelo que incorpora cuatro variables relativas a las estaciones del año (MEST). Los coeficientes obtenidos son muy similares a los de la Tabla 1 comentados anteriormente. El modelo MESTAL incluye la estimación bajo los supuestos de variables latentes con efectos aleatorios.

Al introducir el efecto estacional a través de doce variables (modelo MESTME de la Tabla resumen), una por cada mes del año, los coeficientes obtenidos son nuevamente casi idénticos a los de la Tabla 1 (el modelo MESTMEAL de la Tabla resumen incluye variables latentes con efectos aleatorios).

En algunos artículos se ha destacado la inercia en la demanda de agua. Por ello, en la Tabla resumen se incorporan los resultados de un modelo en el que se incluye un retardo de la variable dependiente (modelo MORE). En ella se puede ver cómo, efectivamente, existe una relación elevada entre el consumo de un mes y el del mes anterior. Aunque se producen variaciones en los coeficientes, se mantienen como significativas todas las variables estudiadas. La misma regresión usando el método generalizado de momentos (MOREG) no supone variaciones significativas en los coeficientes estimados (como variables instrumentales se han utilizado las variables explicativas retardadas y la variable explicada con un retardo de dos periodos).

Tras este análisis econométrico, en la próxima sección se realiza una simulación sobre la incidencia en el bienestar de subidas tarifarias.

\section{INCIDENCIA SOBRE EL BIENESTAR DE LA POLÍTICA TARIFARIA}

Con la política tarifaria se pueden tratar de conseguir diversos objetivos como eficiencia en el consumo, equidad, recuperación íntegra de los costes del servicio o ahorro del recurso. El problema radica en que es difícil conseguir simultáneamente algunos de ellos. Así, por ejemplo, tarifas elevadas sobre el agua ayudan a un uso racional del recurso y fomentan el ahorro. Sin embargo, ello implicará una reducción del bienestar del consumidor derivado del mayor

\footnotetext{
${ }^{10}$ En el modelo MEF se elimina la media de la variable dependiente y de las variables exógenas y se realiza a continuación una nueva regresión habiendo eliminado el efecto de esas variables no observables (Baltagi 2001).
} 
pago por un bien. Además, el hecho de que el agua sea un bien necesario sin sustitutivos, se traduce en una curva de demanda inelástica que implica la necesidad de incrementos relativamente altos de la tarifa para obtener reducciones moderadas del consumo. Estas subidas pueden afectar a sectores de población más desfavorecidos para los que el porcentaje que el gasto en agua supone de su renta es mayor. La discriminación de precios puede ayudar a mitigar o eliminar este problema de equidad pero serían necesarias subvenciones cruzadas (si se quiere respetar el principio de recuperación íntegra de costes) que inciden negativamente en la eficiencia.

Tras el análisis econométrico de la sección anterior se dispone de información con la que simular los efectos de una subida en la tarifa del agua en alta en la cuenca del Segura. Se supondrá que las autoridades se fijan como objetivo la reducción del $10 \%$ del consumo y que el instrumento elegido para ello es la tarifa del agua en alta ${ }^{11}$.

Como indicador de bienestar se utilizará el excedente del consumidor. Si se acepta el supuesto de que los efectos renta serán reducidos debido a que el gasto en agua supone una pequeña parte de la renta del consumidor, las otras dos medidas monetarias de bienestar habitualmente utilizadas en microeconomía, variación compensada y variación equivalente, estarían próximas a la aquí calculada.

Se simularán los efectos para dos escenarios dependiendo de la forma que puede tener la curva de demanda de agua en alta en los municipios de la cuenca del Segura. El primero, en el que la demanda de agua presentaría elasticidad precio constante, y el segundo, caso de una función de demanda lineal.

El supuesto de una curva de demanda con elasticidad precio constante implica que la sensibilidad proporcional del consumo ante cambios en los precios es la misma para precios altos y bajos. Esta forma funcional implicará una disponibilidad al pago elevadísima para las primeras unidades de consumo, lo que está en consonancia con la previsible disponibilidad a pagar por las primeras unidades de un bien vital.

La forma funcional de la curva de demanda en este caso sería:

$$
\mathrm{Q}(\mathrm{P})=\mathrm{AP}^{\varepsilon}
$$

siendo $\mathrm{Q}$ la cantidad demandada y consumida de agua en $\mathrm{m}^{3}$, $\mathrm{P}$ el precio de un $\mathrm{m}^{3}$ de agua, A un parámetro constante y $\varepsilon$ la elasticidad precio de la demanda de agua.

\footnotetext{
${ }^{11}$ Aunque en esta simulación se adopta una subida de la tarifa del agua en alta, habría que destacar que el respeto al principio de recuperación íntegra de costes del servicio hace que la política tarifaria no pueda ser independiente de los costes de suministro. En consonancia con ello, se debería determinar el componente de la tarifa que debe variar y el destino de la recaudación obtenida con ella.
} 
La estimación realizada en la sección anterior indicaría que $\varepsilon$ tomaría el valor de -0,4. Dado el precio del agua en alta y el consumo total de los 42 municipios en el año 2006, podemos calcular el valor de A, que es 79.196.861,81.

Así la función de demanda con elasticidad constante tomaría la forma:

$$
\mathrm{Q}(\mathrm{P})=79.196 .861,81 * \mathrm{P}^{-0.4}
$$

Dado que el objetivo marcado se traduciría en un consumo deseado del $90 \%$ del consumo en 2006, con una simple sustitución se obtendría la tarifa que habría que imponer, 0,4 euros por $\mathrm{m}^{3}$. Con estos dos puntos de la curva de demanda se obtiene la variación del excedente del consumidor que se ilustra en la siguiente figura.

Figura 1

Variación del excedente del consumidor en los 42 municipios de la cuenca del Segura tras la aplicación de una subida tarifaria que redujese el $10 \%$ del consumo de agua. Caso de función de demanda exponencial

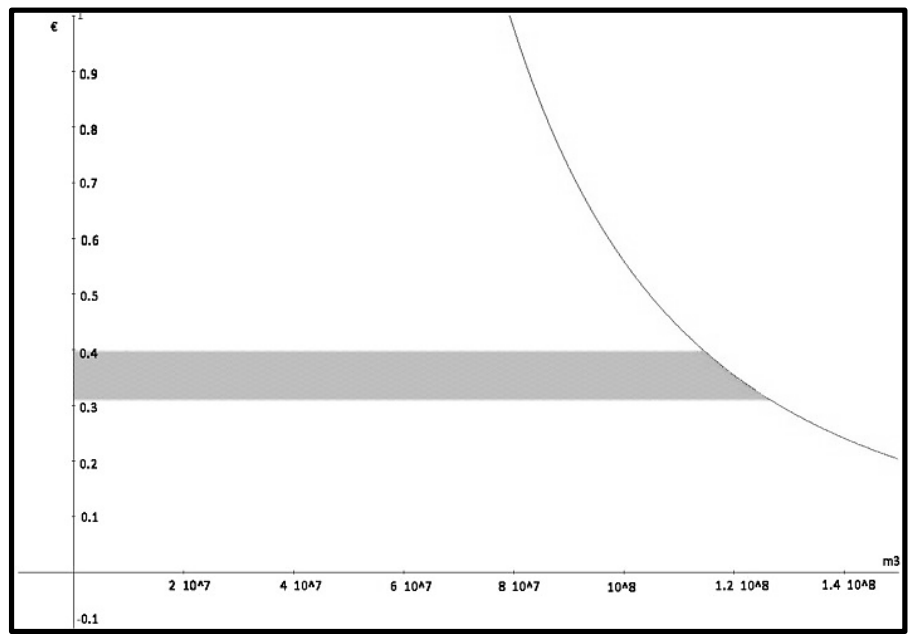

Fuente: Elaboración propia.

Así, la reducción del excedente del consumidor sería de 11.131.760,18 euros para el conjunto de los 42 municipios estudiados. Si dividimos esta cifra total por los 1.310.862 habitantes de los municipios en 2006, se obtendría una reducción de 8,5 euros por persona (y año). El Î́ndice de Precios al Consumo General de la Región de Murcia (IPC en adelante), para 2011 con base 2006, permite valorar esta cifra a 1 de enero de 2012, elevándose a 9,5 euros por persona (y 
año). Si se incluyese la población turística equivalente ${ }^{12}$, la reducción sería de 8 euros que equivalen a 9 euros en enero de 2012.

El supuesto de una curva de demanda lineal tiene varias implicaciones. La primera, que el cambio en la cantidad demandada en respuesta a un precio es el mismo para cualquier nivel de precio. La segunda, que el consumidor es proporcionalmente menos sensible a variaciones del precio a medida que el precio es menor. Finalmente, que existe un precio de reserva a partir del cual la cantidad demandada es cero.

En este caso, el cálculo de la variación del excedente del consumidor podría aproximarse de la siguiente manera. Se aceptaría que la tarifa en alta y el consumo de agua en 2006 son un punto de la curva de demanda de agua. Dado el objetivo de reducción del consumo de agua de un $10 \%$ y el valor de la elasticidad en ese punto de 0,4 se puede obtener la variación porcentual necesaria del precio, que sería de un $25 \%$. Ello nos permitiría obtener otro punto de la función de demanda lineal y calcular de una forma sencilla la variación del excedente del consumidor. La reducción sería de 9.268.168,13 euros para el conjunto de los 42 municipios. Ello se traduce en una reducción de 7 euros por habitante, que actualizados con el IPC, suponen 7,9 euros por habitante (7,5 euros si consideramos la población turística equivalente).

\section{LÍMITES DE LA POLÍTICA TARIFARIA}

Cabe preguntarse si existe algún límite a la efectividad de la política tarifaria para afectar al consumo. En este sentido se incluye a continuación el cálculo de la cantidad de agua consumida que no sería sensible a los incrementos de precio. Para ello se utiliza una función de demanda de tipo Stone-Geary, presentada por Gaudin, Griffin y Sickles (2001). En su estimación intervienen sólo dos parámetros siendo uno de ellos el límite por debajo del cual el consumo no se ve afectado por los precios, al menos a corto plazo. Este parámetro, $\gamma$, se supone que es una función lineal de variables exógenas que se han mostrado significativas en la demanda de agua pero que no están directamente incluidas en la formulación Stone-Geary. Así, para su cálculo se emplea la ecuación

CONSUMOPERCAP $_{i t}=\lambda_{0}+\lambda_{1}$ POBLACIÓN $_{i t}+\lambda_{2}$ VIVSEC $_{i t}+$

$+\lambda_{3}$ PERNOCTACIONES $_{\text {it }}+\lambda_{4}$ TEMPERATURA $_{\text {it }}+$

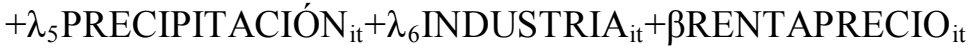

${ }^{12}$ La Memoria de 2006 de la MCT señala que la población de la cuenca se incrementa en 800.000 habitantes en la época estival. Además, señala que el 57\% de la población abastecida corresponde a la provincia de Murcia. Aplicando este mismo porcentaje, ello se traduciría en 456.000 habitantes más para los municipios de la región de Murcia durante los meses de julio y agosto. Traducidos a población equivalente mensual (con una simple división de la cifra anterior por 6), ello supondría 76.000 habitantes más para el conjunto de los 42 municipios. 
con $\mathrm{i}=1 \ldots 42$ y $\mathrm{t}=1 \ldots 84$ y donde CONSUMOPERCAP es el consumo per cápita mensual $\left(\mathrm{m}^{3}\right.$ per cápita) y RENTAPRECIO es el cociente entre las variables renta per cápita mensual del municipio y precio del $\mathrm{m}^{3}$ de agua (deflactado y que es el mismo para todos los municipios). El resto de variables se han definido en el modelo 1. La estimación se presenta en la Tabla 2 que se reproduce a continuación:

Tabla 2

Estimación del modelo [4]

\begin{tabular}{|c|c|c|c|c|}
\hline Variable & Coeficiente & Error estándar & Estadístico t & Prob \\
\hline C & 2.438866 & 0.364941 & 6.682901 & 0.0000 \\
\hline POBLACION & $-5.34 \mathrm{E}-05$ & $3.96 \mathrm{E}-06$ & -13.50746 & 0.0000 \\
\hline VIVSEC & 0.000261 & $1.43 \mathrm{E}-05$ & 18.24879 & 0.0000 \\
\hline PERNOCTACIONES & $-1.25 \mathrm{E}-05$ & $6.99 \mathrm{E}-06$ & -1.785643 & 0.0742 \\
\hline TEMPERATURA & 0.233251 & 0.010074 & 23.15334 & 0.0000 \\
\hline PRECIPITACION & -0.006226 & 0.001990 & -3.129508 & 0.0018 \\
\hline INDUSTRIA & 0.000677 & $7.84 \mathrm{E}-05$ & 8.640509 & 0.0000 \\
\hline RENTAPRECIO & 0.000308 & $2.96 \mathrm{E}-05$ & 10.43315 & 0.0000 \\
\hline \multicolumn{5}{|c|}{ Indicadores de bondad de ajuste } \\
\hline R-cuadrado & 0.330797 & Criterio Akaike & 5.325887 \\
\hline R-cuadrado Ajustado & 0.329466 & Criterio Schwarz & 5.339875 \\
\hline Log verosimilitud & -9386.865 & \multicolumn{5}{|c|}{ F-estadístico } & 248.5699 \\
\hline Estadíst. Durbin-Watson & 0.278760 & \multicolumn{5}{|c|}{ Prob-estadístico) } & 0.000000 \\
\hline
\end{tabular}

Fuente: Elaboración propia.

Usando las estimaciones de los coeficientes $\lambda_{\mathrm{j}}$ y $\beta$ se puede calcular $\gamma$ como $\gamma=(1-\beta)\left(\lambda_{1}\right.$ POBLACIÓN $+\lambda_{2}$ VIVSEC $+\lambda_{3}$ PERNOCTACIONES + $+\lambda_{4}$ TEMPERATURA $+\lambda_{5}$ PRECIPITACIÓN $+\lambda_{6}$ INDUSTRIA)

dónde el subrayado denota la media de la variable en cuestión.

El valor de $\gamma$ es de 4,23 $\mathrm{m}^{3}$. Por tanto, 4,23 $\mathrm{m}^{3}$ indica el límite de consumo de agua en alta per cápita y mes por debajo del cual el precio no es efectivo para influir en el consumo. Dividiendo la cifra anterior por los 30 días del mes, se obtendría un nivel de suministro en alta de 141 litros por habitante y día. La Encuesta sobre el suministro y saneamiento del agua, publicada por el INE, indica que en 2006 el 70\% del agua suministrada en la Región de Murcia se consumió en el hogar (descontadas actividades económicas y consumo municipal). Ello indica que 98,7 litros por habitante y día es la dotación de agua en alta para los hogares que no es sensible al precio. Si se descuenta un $15 \%$ de pérdidas en red, 83,9 litros por habitante y día es el consumo de agua en el hogar que no podría reducirse con incrementos en la tarifa en la Región de Murcia. 
Esta cifra podría interpretarse como un mínimo de consumo de agua necesario, vital, por persona y día. No obstante, dicho valor puede verse afectado por factores tecnológicos (sanitarios y electrodomésticos con menor consumo de agua).

\section{CONCLUSIONES}

En este trabajo se ha estudiado la demanda municipal de agua en alta en la cuenca del Segura. Para ello se han utilizado datos socioeconómicos y de suministro a 42 municipios de la Comunidad Autónoma de la Región de Murcia.

Las conclusiones presentadas a continuación hacen referencia a la citada cuenca pero el modelo es fácil de aplicar a otras con los datos precisos.

La cuenca del Segura es un territorio árido en el que los requerimientos hídricos superan claramente a las disponibilidades, lo que se ha traducido en problemas como la sobreexplotación de los acuíferos y la infradotación de los cultivos de regadío, además de problemas ambientales. Esta situación hace más vulnerable a los territorios de la cuenca puesto que los recursos hídricos son un activo importante para el desarrollo regional, tanto del sector agrícola como del industrial y de los servicios, incluyéndose en este último el turismo.

El abastecimiento urbano de agua en la cuenca del Segura estaría garantizado por ser un uso prioritario. No obstante, el crecimiento futuro del mismo, dada la escasez de este recurso, puede suponer tensiones si ha de hacerse a costa de volúmenes destinados a otros usos. Por ello, se hace cada vez más necesario un adecuado conocimiento de la demanda de agua, así como de su previsible evolución. De otra manera, no será posible una gestión hídrica óptima.

La política tarifaria es una herramienta a través de la cual se puede reducir el consumo de agua si bien tiene un impacto moderado. El valor de la elasticidad precio de la demanda obtenido indica que subidas de las tarifas de un $10 \%$ reducirían el consumo de agua sólo un 4\%. Esta información puede ser relevante en situaciones en las que se está cercano al límite técnico de las infraestructuras. Asimismo, la política tarifaria tiene otra limitación derivada de un mínimo de consumo sobre el que no puede influir. En este artículo se ha calculado que en el hogar, hay un consumo de 84 litros por habitante y día que no podría reducirse con incrementos de las tarifas del agua, al menos a corto plazo. Por ello, si el objetivo es el ahorro del recurso, sería conveniente reforzar esta política con campañas de educación y sensibilización.

Por otro lado, aunque las subidas de tarifas moderan el consumo, también tienen efecto en el bienestar de los ciudadanos al reducir el excedente que el consumo de un bien provee. Las simulaciones realizadas en este artículo señalan que la subida tarifaria necesaria para reducir el consumo de agua un $10 \%$ se traduciría en una pérdida de bienestar de entre 8 y 9,5 euros por habitante (y 
año). Podría ser interesante poner en marcha políticas basadas en incentivos que se tradujeran en reducciones del precio pagado si se alcanzasen reducciones significativas del consumo. Este aspecto sería una línea de investigación futura que complementaría los resultados aquí obtenidos.

El incremento de la renta tiene un mayor impacto en el consumo que el incremento en el precio. Así, según el valor estimado en este modelo, un crecimiento de la renta de un $3 \%$ significaría un incremento de la cantidad demandada de agua del $2,65 \%$. Por tanto, las predicciones de crecimiento económico también han de tenerse en cuenta a la hora de prever la evolución de la demanda urbana de agua. No obstante, es difícil hacer estimaciones rigurosas de la evolución de variables económicas, como el Producto Interior Bruto, en los horizontes de diez o veinte años que se manejan en los Planes Hidrológicos de cuenca.

Los incrementos en la población y el desarrollo turístico tienen un claro impacto en la evolución de la demanda. Las proyecciones de población y los planes urbanísticos de los ayuntamientos proporcionan proxies de la evolución futura de la demanda urbana de agua.

La precipitación apenas consigue reducir el consumo de agua, lo que podría estar relacionado con la estructura de la vivienda, en la que predomina el piso. Cambios hacia urbanizaciones de vivienda con jardín podrían incrementar el consumo y la sensibilidad del mismo a la precipitación. La temperatura tiene un efecto más significativo aunque éste podría no ser lineal. Incrementos de la misma derivados del cambio climático tendrían efecto en el consumo de agua en el hogar (aunque éste no tendría repercusiones tan importantes como las que podría tener sobre la demanda agraria).

La evolución de la demanda industrial de agua merece la realización de un estudio específico. Los factores que influyen en la misma son variados y difíciles prever. No obstante, el impacto que el empleo industrial tendría en el consumo municipal de agua a través de la pequeña industria conectada a redes es moderado, según los resultados obtenidos.

Trabajos como el aquí realizado pueden ayudar a la estimación de la demanda futura de agua. Las predicciones relativas a la demanda urbana suelen realizarse en base a evoluciones previstas de la población. Sin embargo, pueden dejar de lado otros factores cuya influencia, aunque menor, pueda ser también significativa.

Finalmente habría que señalar que los resultados aquí presentados corresponden a un conjunto de cuarenta y dos municipios. Dado que las características de los mismos no son idénticas, una medida general de política tarifaria podría tener reacciones de demanda diferentes en cada uno de ellos. Asimismo, dado que el suministro urbano es competencia municipal, las tarifas finalmente pagadas en los distintos municipios son diferentes con independencia de que el pre- 
cio del agua comprada en alta a la MCT sea el mismo. Por tanto, en ausencia de coordinación, la repercusión en el consumidor final de una subida del precio por parte de la MCT podría ser diferente en cada municipio, y en consecuencia, también la respuesta del consumo de los ciudadanos.

\section{REFERENCIAS BIBLIOGRÁFICAS}

ARBUÉS, F.; GARCÍA VALIÑAS, M.A. y MARTÍNEZ ESPIÑEIRA, R. (2003). "Estimation of residential water demand: a state of the art review". The journal of Socio-Economics 32, pp 81-102.

ARBUÉS, F.; BARBERÁN, R. y VILLANÚA, I. (2004). "Price impact on urban residential water demand: A dynamic panel data approach". Water Resources Research, vol 40, W11402, 10.1029/2004WR003092.

BALTAGI, BADI H. (2001). Econometric Analysis of Panel Data. Second Edition. West Sussex, England: John Wiley and Sons.

BARBERÁN ORTÍ, R.; COSTA TODA, A. y ALEGRE VAL, A. (2008). "Los costes de los servicios urbanos de agua. Un análisis necesario para el establecimiento y control de tarifas". Hacienda Pública Española, 186-(3/2008), pp 123-155.

BEAMONTE CÓRDOBA, E., CASINO MARTÍNEZ, A. y VERES FERRER, E.J. (2010). "Medición de la calidad del agua mediante indicadores. Relación entre éstos y las tarifas de abastecimiento". Estudios de Economía Aplicada, Vol 28-2, pp 357-374.

BELL, D.R. y GRIFFIN, R.C. (2008). "An anual quasidifference apporach to water price elasticity". Water Resources Research, 44, W08420, doi:10.1029/2007WR006233.

BELL, D.R. y GRIFFIN, R.C. (2011). "Urban water demand with periodic error correction". Land Economics 87(3), pp 528-544.

CARTER, D.W. y MILON, J.W. (2005). "Price knowledge in household demand for utility services". Land Economics 81 (2), pp 265-283.

CONFEDERACIÓN HIDROGRÁFICA DEL SEGURA (1998). Plan Hidrológico de la Cuenca del Segura.

DEL VILLAR, A. (2010). "Los precios de los servicios del agua. Un análisis prospectivo de demanda sobre los usos domésticos". Estudios de Economía Aplicada, Vol 28-2, pp 333-356.

GARCÍA VALIÑAS, M.A. (2005). "Fijación de precios para el servicio municipal de suministro de agua: un ejercicio de análisis de bienestar". Hacienda Pública Española, 172-1, pp 119-142. 
GAUDIN, S., GRIFFIN, R.C. y SICKLES, R.C. (2001). "Demand especification for municipal water management: evaluation of the Stone-Geary form". Land Economics 77 (3), pp 399-422.

GÓMEZ-LIMÓN, J.A. y MARTÍN-ORTEGA, J. (2011). "Agua, economía y territorio: nuevos enfoques de la Directiva Marco del Agua para la gestión del recurso". Estudios de Economía Aplicada, vol. 29-1, pp 65-94.

GONZÁLEZ GÓMEZ, F. y GARCÍA RUBIO, M.A. (2008). "Efficiency in the management of urban wáter services. What have we learned after four decades of research?" Hacienda Pública Española, 185-(2/2008), pp 39-67.

INSTITUTO NACIONAL DE ESTADÍSTICA. Encuesta de suministro y tratamiento de agua.

MANCOMUNIDAD DE LOS CANALES DEL TAIBILLA (2007). La gestión del servicio 2006.

MARTÍNEZ ESPIÑEIRA, R. (2003). "Estimating Water Demand under Increasing-Block Tariffs Using Aggregate Data and Proportions of Users per Block". Environmental and Resource Economics, 26, pp 5-23.

MARTÍNEZ ESPIÑEIRA, R. (2007). "An estimation of residential water demand using co-integration and error correction techniques". Journal of Applied Economics 10 (1), pp 161-184.

NORDIN, J.A. (1976). "A Proposed Modification on Taylor's Demand-Supply Analysis: Comment". The Bell Journal of Economics 7(2), pp 719-721.

NOVALES CINCA, ALFONSO (1993). Econometría. Segunda Edición. Editorial McGraw-Hill.

OLMSTEAD, S.M., HANEMANN, W.M. y STAVINS, R.N. (2007). "Water demand under alternative price structures". Journal of Environmental Economics and Management 54, pp 181-198.

PÉREZ BLANCO, C.D., GÓMEZ GÓMEZ, C.M. y GARRIDO YSERTE, R. (2010). "Cambio estructural regional y agua: escasez, dependencia e impactos sobre el tejido económico: El caso de Andalucía. Estudios de Economía Aplicada, Vol 28-2, pp 423-446.

SEVILLA JIMÉNEZ, M., TORREGROSA MARTÍ, T. y MORENO, L. (2010). "Un panorama sobre la economía del agua". Estudios de Economía Aplicada, Vol 28-2, pp 265-304.

TAYLOR, R.G., MCKEAN, J.R. y YOUNG, R.A. (2004). "Alternate price specifications for estimating residential water demand with fixed fees". Land Economics 80 (3), pp 463-475.

TRUJILLO, L. (1994). "Fijación de precios óptimos en el suministro urbano de agua". Revista de Economía Aplicada 5 (vol.II), pp 111-135. 


\section{ANEXO}

Tabla Resumen

Estimaciones de modelos

\begin{tabular}{|c|c|c|c|c|}
\hline & \multicolumn{2}{|c|}{ MODELO [1] con var. POBLA25 } & \multicolumn{2}{|c|}{ MODELO MEAL } \\
\hline Variable & Coeficiente & Estadístico t & Coeficiente & Estadístico $\mathrm{t}$ \\
\hline $\mathrm{C}$ & -3.215336 & -7.476867 & 1.720626 & 4.444676 \\
\hline PRECIO & -0.419443 & -5.009950 & -0.091450 & -2.112139 \\
\hline RENTA & 0.903885 & 18.84849 & 0.396810 & 8.633379 \\
\hline POBLACION & 0.652784 & 35.43457 & 0.550564 & 12.33570 \\
\hline VIVSEC & 0.040443 & 7.738002 & 0.046056 & 1.561319 \\
\hline PERNOCTACIONES & 0.042615 & 5.486617 & 0.128344 & 11.91046 \\
\hline TEMPERATURA & 0.268591 & 13.76722 & 0.284197 & 28.68955 \\
\hline PRECIPITACION & -0.020785 & -8.595087 & -0.011642 & -10.65095 \\
\hline INDUSTRIA & 0.112863 & 11.98331 & 0.003995 & 0.407521 \\
\hline \multirow[t]{2}{*}{ POBLA25 } & -0.142642 & -1.525960 & - & - \\
\hline & \multicolumn{2}{|c|}{ MODELO MEF } & \multicolumn{2}{|c|}{ MODELO MEST } \\
\hline Variable & Coeficiente & Estadístico $\mathrm{t}$ & Coeficiente & Estadístico $\mathrm{t}$ \\
\hline $\mathrm{C}$ & 4.072378 & 8.234699 & - & - \\
\hline PRECIO & -0.049429 & -1.076874 & -0.437506 & -5.260793 \\
\hline RENTA & 0.518961 & 8.870567 & 0.918456 & 19.66756 \\
\hline POBLACION & 0.175572 & 2.157996 & 0.650316 & 36.80264 \\
\hline VIVSEC & 0.128255 & 1.104471 & 0.042790 & 8.267032 \\
\hline PERNOCTACIONES & 0.128842 & 11.65440 & 0.043451 & 5.777961 \\
\hline TEMPERATURA & 0.285023 & 28.57954 & -0.017382 & -6.531150 \\
\hline PRECIPITACION & -0.011339 & -10.36409 & 0.096696 & 2.672995 \\
\hline \multirow[t]{2}{*}{ INDUSTRIA } & -0.013087 & -1.300641 & 0.107462 & 11.51933 \\
\hline & \multicolumn{2}{|c|}{ MODELO MESTAL } & \multicolumn{2}{|c|}{ MODELO MESTME } \\
\hline Variable & Coeficiente & Estadístico $\mathrm{t}$ & Coeficiente & Estadístico $t$ \\
\hline $\mathrm{C}$ & 2.299103 & 6.026708 & -0.490990 & -5.933556 \\
\hline PRECIO & -0.069886 & -1.656854 & 0.964833 & 20.61382 \\
\hline RENTA & 0.358032 & 7.965772 & 0.656206 & 37.41055 \\
\hline POBLACION & 0.538402 & 12.20749 & 0.044670 & 8.694936 \\
\hline VIVSEC & 0.048450 & 1.647416 & 0.040866 & 5.460726 \\
\hline PERNOCTACIONES & 0.132563 & 12.35510 & -0.022751 & -7.949052 \\
\hline TEMPERATURA & -0.005547 & -4.706516 & -0.160310 & -3.007659 \\
\hline PRECIPITACION & 0.191612 & 11.24363 & 0.102560 & 11.05862 \\
\hline \multirow[t]{2}{*}{ INDUSTRIA } & 0.003391 & 0.355179 & -0.490990 & -5.933556 \\
\hline & \multicolumn{2}{|c|}{ MODELO MESTMEAL } & \multicolumn{2}{|c|}{-} \\
\hline Variable & Coeficiente & Estadístico t & - & - \\
\hline $\mathrm{C}$ & 2.694319 & 7.148313 & & \\
\hline PRECIO & -0.079452 & -1.934151 & & \\
\hline RENTA & 0.360248 & 8.202556 & & \\
\hline POBLACION & 0.536210 & 12.30643 & & \\
\hline VIVSEC & 0.055557 & 1.892414 & & \\
\hline PERNOCTACIONES & 0.123202 & 11.11898 & & \\
\hline TEMPERATURA & -0.007620 & -6.089833 & & \\
\hline PRECIPITACION & 0.046686 & 1.704304 & & \\
\hline INDUSTRIA & 0.003231 & 0.347743 & & \\
\hline
\end{tabular}


Tabla Resumen (continuación)

Estimaciones de modelos

\begin{tabular}{|c|c|c|c|c|}
\hline & \multicolumn{2}{|c|}{ MODELO MORE } & \multicolumn{2}{c|}{ MODELO MOREG } \\
\hline Variable & Coeficiente & Estadístico t & Coeficiente & Estadístico t \\
\hline C & -0.402789 & -2.885182 & -0.230946 & -1.331505 \\
\hline CONSUMO (-1) & 0.895765 & 141.5748 & 0.898340 & 116.9128 \\
\hline PRECIO & -0.064796 & -2.015862 & -0.058079 & -1.607384 \\
\hline RENTA & 0.098838 & 5.236422 & 0.108448 & 5.418454 \\
\hline POBLACION & 0.064280 & 8.048606 & 0.066917 & 7.738960 \\
\hline VIVSEC & 0.003549 & 1.750849 & 0.001500 & 0.400595 \\
\hline PERNOCTACIONES & 0.004942 & 1.685700 & 0.004949 & 2.285544 \\
\hline TEMPERATURA & -0.012761 & -13.69022 & -0.009768 & -1.156180 \\
\hline PRECIPITACION & 0.054199 & 6.945148 & -0.036677 & -1.559398 \\
\hline INDUSTRIA & 0.012933 & 3.511810 & 0.010779 & 2.604241 \\
\hline
\end{tabular}

Fuente: Elaboración propia. 